\title{
Randomised controlled trial of a short course of traditional acupuncture compared with usual care for persistent non-specific low back pain
}

\author{
K J Thomas, H MacPherson, L Thorpe, J Brazier, M Fitter, M J Campbell, M Roman, S J Walters, J Nicholl
}

\begin{abstract}
Objective To determine whether a short course of traditional acupuncture improves longer term outcomes for patients with persistent non-specific low back pain in primary care.

Design Pragmatic, open, randomised controlled trial.

Setting Three private acupuncture clinics and 18 general practices in York, England.

Participants 241 adults aged 18-65 with non-specific low back pain of 4-52 weeks' duration.

Interventions 10 individualised acupuncture treatments from one of six qualified acupuncturists (160 patients) or usual care only (81 patients).

Main outcome measures The primary outcome was SF-36 bodily pain, measured at 12 and 24 months. Other outcomes included reported use of analgesics, scores on the Oswestry pain disability index, safety, and patient satisfaction.

Results 39 general practitioners referred 289 patients of whom 241 were randomised. At 12 months average SF-36 pain scores increased by 33.2 to 64.0 in the acupuncture group and by 27.9 to 58.3 in the control group. Adjusting for baseline score and for any clustering by acupuncturist, the estimated intervention effect was 5.6 points $(95 \%$ confidence interval -0.2 to 11.4$)$ at 12 months $(\mathrm{n}=213)$ and 8.0 points (2.8 to 13.2$)$ at 24 months $(\mathrm{n}=182)$. The magnitude of the difference between the groups was about $10 \%-15 \%$ of the final pain score in the control group. Functional disability was not improved. No serious or life threatening events were reported.

Conclusions Weak evidence was found of an effect of acupuncture on persistent non-specific low back pain at 12 months, but stronger evidence of a small benefit at 24 months. Referral to a qualified traditional acupuncturist for a short course of treatment seems safe and acceptable to patients with low back pain.

Trial registration ISRCTN80764175.
\end{abstract}

\section{Introduction}

Non-specific low back pain is typically intermittent and recurrent and associated with high health, social, and economic costs. About $16 \%$ of the adult population in the United Kingdom consult their general practitioner yearly for back pain, and most episodes are managed in primary care with drugs or other non-surgical treatments. ${ }^{12}$ Cochrane reviews exist for 13 different non-surgical treatments for back pain, including acupuncture, but the evidence is largely inconclusive and the best way to manage simple low back pain remains unclear. ${ }^{34}$ For chronic low back pain there is evidence of short term pain relief and functional improvement using acupuncture compared with no treatment or sham therapy, but evidence for long term effectiveness is sparse. ${ }^{5}$

Acupuncture is used by an estimated $2 \%$ of adults in the United Kingdom each year for a range of conditions, including back pain. Less than $10 \%$ of provision is through the United Kingdom's health service, mostly in primary care. ${ }^{6}$ The largest single group of professional acupuncturists in the United Kingdom are registered with the British Acupuncture Council, which requires three years' training or equivalent, and these acupuncturists practise traditional acupuncture based on the principles of Chinese medicine.

We determined whether referral to a short course of traditional acupuncture delivered in a non-NHS setting improves longer term outcomes for patients with non-specific low back pain in primary care. We also monitored safety and acceptability of acupuncture to patients. A cost effectiveness study was carried out alongside this pragmatic, open, randomised controlled trial. ${ }^{8}$

\section{Participants and methods}

We recruited to our study patients aged 18-65 with non-specific low back pain of 4-52 weeks' duration who were assessed as suitable by their general practitioner for primary care management. We excluded patients who were receiving current acupuncture treatment, who had possible spinal disease (for example, carcinoma), severe or progressive motor weakness, prolapsed central disc, past spinal surgery, bleeding disorders (for example, haemophilia), or pending litigation. Thirty nine general practitioners from 16 practices identified suitable patients at consultation. The study researcher (LT) assessed eligibility, obtained consent, and recruited patients to the trial.

\section{Outcome measures}

Our primary outcome was the bodily pain dimension of the SF-36 at 12 months, scored on a 0 to 100 (no pain) scale. This assesses pain experienced in the past four weeks and has been validated for use in primary care and in patients with back pain. ${ }^{9-11}$ After a progress review and analysis of partial data for three months, we amended the protocol to include economic data at 24 months, along with an additional clinical end point at 24 months.

Our secondary outcomes were scores on the Oswestry pain disability index, ${ }^{12}$ the McGill present pain index,${ }^{13}$ and the seven remaining dimensions of the SF-36 health status questionnaire. ${ }^{9}$ 
Each was administered at baseline and at 3, 12, and 24 months. Satisfaction with treatment was assessed at three months. ${ }^{14}$ We included questions on concerns about back pain ${ }^{14}$ and current use of analgesics at 12 and 24 months. At 24 months we collected patient reports of back pain in the past year. Follow-up was carried out by post; when this was unsuccessful we sought main outcome data by telephone.

We assessed safety by monitoring adverse events reported by patients at each acupuncture session, and at follow-up of those who did not complete treatment. We also collected standardised information on responses to treatment directly from patients. Acceptability was assessed through the uptake of acupuncture, dropout rates, and patient completed items on satisfaction with aspects of care and willingness to try acupuncture again.

\section{Intervention}

We randomised patients to receive either a short course of traditional acupuncture or usual care only. The six participating acupuncturists were registered with the British Acupuncture Council, and each had at least three years' experience. The first appointments were held in one of two non-NHS clinics in York, usually within two weeks of randomisation. Acupuncture care comprised up to 10 individualised treatment sessions over three months. Acupuncturists determined the content and the number of treatments according to patients' needs. The acupuncture needles were disposable. All patients remained under the care of their general practitioner.

Patients in the usual care group received NHS treatment according to their general practitioner's assessment of need. We collected information from patients at 3, 12, and 24 months on treatments received for low back pain.

\section{Randomisation and blinding}

The study statistician (MJC) provided a computer generated, blocked randomisation sequence. After consent and baseline measures had been obtained, the study researcher (LT) was told the individual treatment assignments by telephone. The number of cases in each randomisation block was not revealed to the researcher. Allocation to practitioner was by availability of appointments and convenience to patients. As this was an open trial, neither participants nor researchers were blind to treatment assignment. However, analysis of the primary outcome was repeated by a second statistician (SJW) who was blind to treatment allocation.

\section{Statistical analysis}

On the basis of a pilot study, ${ }^{15}$ and allowing for a $10 \%-15 \%$ dropout rate, we determined that we needed 100 patients in each arm to detect a difference in outcome between the groups of 10 (SD 19.3) points for the SF-36 pain score at 12 months, at $90 \%$ power and a two sided $5 \%$ significance level. The number of patients was subsequently increased to 240 , retaining the original power but with a 2:1 randomisation to the acupuncture group to allow for effects between acupuncturists to be tested. Data were analysed using SPSS version 11.5 and Stata version 8. Analysis was undertaken on an intention to treat basis, using analysis of covariance for outcomes at 12 and 24 months, with baseline SF-36 pain scores as a covariate. We undertook a complete case analysis after exploration of known characteristics. The adjusted estimated effect and 95\% confidence intervals are reported for all results.

Clustering of intervention patients by acupuncturist may inflate the standard error of the treatment comparison. ${ }^{16}$ We estimated clustering by acupuncturist using robust standard errors, which allows for separate estimates of variance for each patient in the control group and a shared variance for each cluster of patients assigned to a particular acupuncturist. We undertook a sensitivity analysis using a more complex regression model comprising baseline covariates that were observed to affect outcomes at three months, and by undertaking analysis using last observation carried forward to deal with missing values. We carried out exploratory analysis using analysis of covariance to examine the interaction between the effect of acupuncture and two variables: patients' expectations of improvement to low back pain and patients' belief that acupuncture might help, assessed before randomisation.

\section{Results}

Overall, 241 patients were recruited to the trial between August 1999 and January 200l. Two patients subsequently withdrew (fig 1). Table 1 shows the baseline personal and clinical characteristics of patients in each group. Both groups had on average recurrent episodes of back pain for four months and about half the patients reported more than five previous episodes of back pain. With the exception of inability to work because of low back pain, no significant differences were observed between the groups at baseline.

\section{Treatments}

All patients randomised to acupuncture accepted the referral. Pain resolved in nine patients before treatment; the remaining patients received an average of 8.1 treatments. Adjunctive care received from acupuncturists or general practitioners included massage and advice on diet, rest, and exercise (fig 2). Details of the acupuncture treatments are published elsewhere. ${ }^{17}$

Usual care comprised a mix of interventions. Half the patients randomised to usual care received physiotherapy or manipulation during the first three months. Other interventions included drugs and recommended back exercises (fig 2).

\section{Follow-up}

The dropout rate was within the range expected at 12 months. The rate was higher at 24 months, but similar in both groups. Those lost to follow-up were younger and had poorer SF-36 pain scores at three months. This pattern was observed in both groups, indicating no evidence of a difference between the groups in those lost to follow-up. ${ }^{18}$

\section{Clinical outcomes}

Table 2 shows pain scores for patients completing follow-up at 12 and 24 months. Table 3 shows the adjusted differences between groups. An intervention effect of 5.6 points (95\% confidence interval -0.2 to 11.4 ) was found on the primary outcome SF-36 pain dimension in favour of the acupuncture care group at 12 months. At 24 months a statistically significant difference was found between the groups, with an estimated effect of 8.0 points (2.8 to 13.2). The acupuncture group had better scores on the Oswestry pain disability index and the McGill present pain intensity measure. These differences failed to reach statistical significance in most analyses (table 3).

\section{Patient perceived treatment benefits}

At three months patients in the acupuncture group were significantly more likely to be "very satisfied" with their treatment and with their overall care compared with patients receiving usual care but showed no such difference in satisfaction with information received. At 24 months the acupuncture group were more likely to report fewer concerns about their back pain, less likely to report current use of analgesics for their pain, and more likely to report no pain for the past 12 months (table 4). 


\section{Adverse events}

No serious adverse events (an event resulting in admission to hospital or permanent disability, or both, or death) were reported by patients in the acupuncture care group. Four of the 16 patients who withdrew from acupuncture care mentioned specific minor adverse events, including transient pain at the site of needling. Of the patients who received at least one acupuncture treatment and who provided data at three months, $23 \%(30 / 133)$ reported a temporary worsening of low back pain during the treatment phase that bothered them "a lot" or "a great deal" at the time. However, 86\% (112/133) experienced acupuncture treatment as relaxing, and 91\% (126/139) were willing to have acupuncture again.

\section{Prior expectations and beliefs}

Comparison of groups classified according to expectations of improvement in back pain and belief that acupuncture might help, both stated before randomisation, showed evidence that expectations of improvement had a positive treatment effect, and weak evidence of an interaction effect whereby positive belief was associated with less benefit than neutral belief. (table 5).

\section{Discussion}

Weak evidence was found of an effect of acupuncture care on non-specific low back pain at 12 months, but stronger evidence of a small benefit at 24 months.

On the basis of pilot data our study was designed to detect a larger difference of 10 points, which was not achieved at either 12 or 24 months. A difference of at least five points in the mean score of the SF-36 bodily pain dimension is, however, considered to represent a clinically worthwhile benefit ${ }^{9}$ and a difference of between five and nine points can be viewed as a moderate
Table 1 Baseline characteristics of patients with non-specific low back pain who were randomised to acupuncture care or to usual care only. Values are numbers (percentages) unless stated otherwise

\begin{tabular}{|c|c|c|}
\hline Characteristic & $\begin{array}{l}\text { Acupuncture care } \\
\quad(n=159)\end{array}$ & Usual care $(n=80)$ \\
\hline Mean (SD) age (years) & $42.0(10.8)$ & $44.0(10.4)$ \\
\hline Range & 20 to 64 & 26 to 64 \\
\hline Mean (SD) weeks with back pain, range & 17.1 (13.5), 4-48 & 16.7 (14.6), 4-48 \\
\hline Mean (SD) SF-36 physical functioning score & $55.5(25.2)$ & $60.0(21.8)$ \\
\hline Male & $60(38)$ & $34(43)$ \\
\hline Female & $99(62)$ & $46(58)$ \\
\hline \multicolumn{3}{|l|}{ Work status: } \\
\hline Works full time & $82(52)$ & $45(56)$ \\
\hline Works part-time & $40(25)$ & $22(28)$ \\
\hline Housewife & $13(8)$ & $7(9)$ \\
\hline Retired & $7(4)$ & $4(5)$ \\
\hline Student & $3(2)$ & $1(1)$ \\
\hline $\begin{array}{l}\text { Permanently unable to work owing to low } \\
\text { back pain }\end{array}$ & $11(7)$ & - \\
\hline $\begin{array}{l}\text { Permanently unable to work for other } \\
\text { health reason }\end{array}$ & $3(2)$ & $1(1)$ \\
\hline \multicolumn{3}{|l|}{ No of previous episodes of low back pain:* } \\
\hline None & $25(16)$ & $13(16)$ \\
\hline $1-5$ & $57(36)$ & $23(29)$ \\
\hline$>5$ & $77(48)$ & $44(55)$ \\
\hline Presence of leg pain & $106(67)$ & $59(74)$ \\
\hline \multicolumn{3}{|l|}{ Expectation of back pain in six months: } \\
\hline Better & $80(50)$ & $30(38)$ \\
\hline Same or worse & $78(49)$ & $49(61)$ \\
\hline Don’t know & $1(1)$ & $1(1)$ \\
\hline Thinks acupuncture will help with back pain & $111(70)$ & $51(64)$ \\
\hline Drugs for low back pain in past four weeks & $140(88)$ & $72(90)$ \\
\hline $\begin{array}{l}\text { Major health problems in addition to back } \\
\text { pain }\end{array}$ & 44 (28) & $25(31)$ \\
\hline Ever used private acupuncture for any reason & $20(13)$ & $7(9)$ \\
\hline
\end{tabular}

*Period of low back pain separated by at least three months of being pain free. 


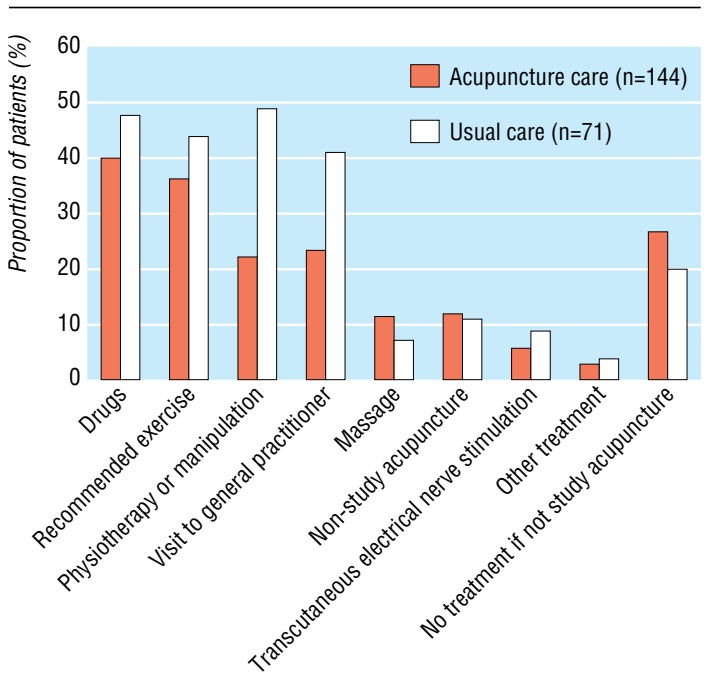

Fig 2 Proportion of patients reporting at least one treatment (0 to 12 weeks) by treatment and allocation group

effect. ${ }^{19}{ }^{20}$ In this study the magnitude of the effect extends to eight points at 24 months, a difference between groups of about $10 \%-15 \%$ of the final pain score in the control group, and achieves statistical significance. We found no evidence of functional improvement. Other patient relevant outcomes included patient satisfaction with acupuncture care, reduced concerns about back pain, and reduced use of analgesics.
Table 4 Other outcomes reported at 24 months' follow-up after acupuncture care or usual care. Values are numbers (percentages) unless stated otherwise

\begin{tabular}{|c|c|c|c|c|}
\hline Outcome & $\begin{array}{l}\text { Acupuncture } \\
\text { group }\end{array}$ & $\begin{array}{l}\text { Usual care } \\
\text { group }\end{array}$ & $\begin{array}{l}\% \text { Difference } \\
(95 \% \mathrm{Cl})\end{array}$ & $\begin{array}{c}\mathbf{P} \\
\text { value }^{*}\end{array}$ \\
\hline \multicolumn{5}{|l|}{$\begin{array}{l}\text { In the past } 12 \text { months have you } \\
\text { had low back pain or leg pain?: }\end{array}$} \\
\hline Yes & $93(82)$ & $46(92)$ & \multirow{2}{*}{$\begin{array}{c}-10 \\
(-20 \text { to } 2)\end{array}$} & \multirow{2}{*}{$0.06+$} \\
\hline No & $21(18)$ & $4(8)$ & & \\
\hline \multicolumn{5}{|l|}{$\begin{array}{l}\text { During the past four weeks have } \\
\text { you used any medicine for low } \\
\text { back pain?: }\end{array}$} \\
\hline Yes & $45(40)$ & 29 (59) & \multirow{2}{*}{$\begin{array}{c}-19 \\
(-35 \text { to }-3)\end{array}$} & \multirow{2}{*}{$0.03 \dagger$} \\
\hline No & $68(60)$ & $20(41)$ & & \\
\hline \multicolumn{5}{|l|}{$\begin{array}{l}\text { Compared with the worry you felt } \\
12 \text { month ago, how worried are } \\
\text { you about your back pain } \\
\text { problem now?: }\end{array}$} \\
\hline Much less or less worried & $79(71)$ & $19(39)$ & \multirow{3}{*}{32 (15 to 46$)$} & \multirow{3}{*}{$<0.001 \ddagger$} \\
\hline Same & $22(20)$ & $23(47)$ & & \\
\hline More or much more worried & $11(10)$ & $7(14)$ & & \\
\hline
\end{tabular}

One limitation of our study is the possibility of an effect of clustering by practitioner (six acupuncturists delivered the treatment) on the statistical significance of the outcome. In this case, cluster analysis made little difference to the primary outcome of bodily pain at 12 and 24 months. Sensitivity analysis taking into account baseline covariates increased the intervention effect but did not alter the main results. Groups were well balanced at base-

\begin{tabular}{|c|c|c|c|c|c|c|c|c|c|c|c|c|}
\hline \multirow{3}{*}{ Variable } & \multicolumn{4}{|c|}{ Baseline } & \multicolumn{4}{|c|}{12 months } & \multicolumn{4}{|c|}{24 months } \\
\hline & \multicolumn{2}{|c|}{ Acupuncture care } & \multicolumn{2}{|c|}{ Usual care } & \multicolumn{2}{|c|}{ Acupuncture care } & \multicolumn{2}{|c|}{ Usual care } & \multicolumn{2}{|c|}{ Acupuncture care } & \multicolumn{2}{|c|}{ Usual care } \\
\hline & Mean (SD) & $\begin{array}{c}\text { No of } \\
\text { patients }\end{array}$ & Mean (SD) & $\begin{array}{c}\text { No of } \\
\text { patients }\end{array}$ & Mean (SD) & $\begin{array}{l}\text { No of } \\
\text { patients }\end{array}$ & Mean (SD) & $\begin{array}{l}\text { No of } \\
\text { patients }\end{array}$ & Mean (SD) & $\begin{array}{c}\mathrm{N} \text { of } \\
\text { patients }\end{array}$ & Mean (SD) & $\begin{array}{l}\text { No of } \\
\text { patients }\end{array}$ \\
\hline \multicolumn{13}{|l|}{ All cases: } \\
\hline $\begin{array}{l}\text { SF-36 bodily } \\
\text { pain* }^{\star}\end{array}$ & $30.8(16.2)$ & 159 & $30.4(18.0)$ & 80 & $64.0(25.6)$ & 147 & $58.3(22.2)$ & 68 & $67.8(24.1)$ & 123 & $59.5(23.4)$ & 59 \\
\hline $\begin{array}{l}\text { Oswestry pain } \\
\text { disability } \\
\text { indext }\end{array}$ & $33.7(15.4)$ & 159 & $31.4(14.2)$ & 80 & $20.6(19.3)$ & 134 & $19.6(15.4)$ & 57 & $18.3(16.5)$ & 114 & $21.0(14.2)$ & 50 \\
\hline $\begin{array}{l}\text { McGill present } \\
\text { pain indexf }\end{array}$ & $2.64(1.0)$ & 159 & $2.70(1.0)$ & 80 & $1.43(1.1)$ & 135 & $1.53(0.9)$ & 57 & $1.42(1.1)$ & 113 & $1.71(1.1)$ & 49 \\
\hline
\end{tabular}

${ }^{*}$ Scored on a 0 to 100 (no pain) scale.

tScored on a 0 (no pain) to 100 scale.

fScored on a 0 (no pain) to 5 scale.

Table 3 Difference in outcome scores between acupuncture and usual care groups at 12 and 24 months

\begin{tabular}{|c|c|c|c|c|c|c|}
\hline \multirow{2}{*}{ Variable } & \multicolumn{3}{|c|}{12 months } & \multicolumn{3}{|c|}{24 months } \\
\hline & No of patients & Difference $^{\star}(95 \% \mathrm{CI})$ & $P$ value & No of patients & 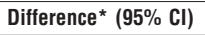 & $P$ value \\
\hline \multicolumn{7}{|l|}{ SF-36 bodily pain score: } \\
\hline Unadjusted difference & 215 & $5.7(-1.4$ to 12.8$)$ & 0.11 & 182 & 8.2 (0.8 to 15.7$)$ & 0.031 \\
\hline $\begin{array}{l}\text { Difference adjusted for baseline pain score and } \\
\text { clustering by acupuncturist }\end{array}$ & 213 & $5.6(-0.2$ to 11.4$)$ & 0.06 & 182 & 8.0 (2.8. to 13.2) & 0.003 \\
\hline $\begin{array}{l}\text { Difference adjusted for baseline pain score and } \\
\text { other baseline covariates } \dagger\end{array}$ & 212 & $6.0(-0.6$ to 12.6$)$ & 0.07 & 179 & 9.0 (1.8. to 16.2$)$ & 0.015 \\
\hline \multicolumn{7}{|l|}{ Oswestry pain disability index: } \\
\hline Unadjusted scores & 191 & $0.9(-3.8$ to 5.8$)$ & 0.68 & 164 & $-2.7(-7.1$ to 1.6$)$ & 0.22 \\
\hline $\begin{array}{l}\text { Difference adjusted for baseline pain score and } \\
\text { clustering by acupuncturist }\end{array}$ & 191 & $-0.5(-5.1$ to 4.2$)$ & 0.85 & 164 & $-3.4(-7.8$ to 1.0$)$ & 0.21 \\
\hline \multicolumn{7}{|l|}{ McGill present pain index: } \\
\hline Unadjusted scores & 192 & $-0.1(-0.4$ to 0.2$)$ & 0.55 & 162 & $-0.3(-0.7$ to 0.1$)$ & 0.1 \\
\hline $\begin{array}{l}\text { Difference adjusted for baseline pain score and } \\
\text { clustering by acupuncturist }\end{array}$ & 192 & $-0.1(-0.4$ to 0.3$)$ & 0.67 & 162 & $-0.2(-0.6$ to 0.1$)$ & 0.21 \\
\hline
\end{tabular}


Table 5 Exploratory analyses of baseline expectations of improvement to back pain and belief that acupuncture might help, with SF-36 pain scores at 24 months' follow-up after acupuncture care or usual care

\begin{tabular}{|c|c|c|c|c|c|}
\hline \multirow{2}{*}{ Baseline question } & \multirow{2}{*}{ No of patients } & \multicolumn{2}{|c|}{ Mean scores for SF-36 bodily pain at 24 months } & \multirow{2}{*}{$\begin{array}{l}\text { Estimated treatment effect adjusted } \\
\text { for baseline pain score }\end{array}$} & \multirow{2}{*}{$P$ value for interactior } \\
\hline & & Acupuncture care & Usual care & & \\
\hline \multicolumn{6}{|c|}{$\begin{array}{l}\text { How do you expect your back pain to be in six } \\
\text { months? }\end{array}$} \\
\hline Much better or better & 82 & 73.1 & 56.9 & 16.7 & 0.04 \\
\hline Same or worse & 97 & 62.7 & 60.6 & 1.2 & \\
\hline \multicolumn{6}{|c|}{$\begin{array}{l}\text { Do you think your back problem may be helped } \\
\text { by acupuncture? }\end{array}$} \\
\hline Yes & 128 & 66.4 & 62.8 & 3.3 & 0.07 \\
\hline Don't know or no* & 56 & 71.0 & 52.6 & 18.2 & \\
\hline
\end{tabular}

*Only one patient stated "no."

line with the exception of 11 patients who described themselves as permanently unable to work because of low back pain, all of whom were in the acupuncture arm. Excluding these patients increased the estimated effect of acupuncture at 12 months. ${ }^{18}$

Secondary outcome measures showed mixed effects. Those measuring the effect of pain on daily living did not show a significant acupuncture effect at 12 or 24 months. Detailed analysis of item responses to the SF-36 and Oswestry pain disability index could help to identify the specific effect of acupuncture on low back pain. Patients in the acupuncture group reported a substantial reduction in concerns about their back pain that was not observed in the usual care group. A qualitative investigation is indicated to explore the meaning to patients of this reduction in worry, its relation to patient coping strategies, and its implications for the care and management of this group of patients.

We evaluated a package of care and cannot isolate the components of acupuncture treatment that are associated with the outcomes observed in the acupuncture group. Beyond the needling itself, several aspects of acupuncture care in this trial could contribute to its observed effectiveness, including the individualising of treatment, ${ }^{21}$ the interaction effect generated by the combination of specific effects, ${ }^{22}$ the practitioner's skills at developing good therapeutic relationships, ${ }^{23}$ process benefits such as protected time and attention from the practitioner, ${ }^{24}$ and the widely reported relaxing experience of the treatment itself. ${ }^{25}$

An open pragmatic trial avoids the potential problems associated with using sham acupuncture as the control. ${ }^{26}$ Such a design may, however, be vulnerable to confounding or bias owing to prior patient beliefs about how acupuncture might help, especially when using subjectively assessed outcome measures, such as perceived pain. Positive patient beliefs about acupuncture have been cited as a possible mechanism for non-specific effects observed in acupuncture trials. ${ }^{27} 28$ Our exploratory analysis does not seem to support this hypothesis. Patients in the acupuncture group with a prior positive belief in the effectiveness of acupuncture fared little better than those randomised to usual care. In contrast, patients with neutral prior belief gained relatively more benefit from acupuncture care. In addition, positive expectations of improvement in back pain seem to reinforce the effect of acupuncture care.

Overall, $76 \%$ of our participants were followed up at 24 months. We detected no difference between groups in the known characteristics of missing cases, and sensitivity analysis substituting missing values with prior observations did not change the main findings, but the effect of the missing data is unknown. The strengths of our study were the pragmatic randomised design, successful patient recruitment, wide eligibility criteria, and a non-restrictive treatment protocol that allowed acupuncturists to treat patients as they would do in everyday practice..$^{29}$ The acupuncturists were selected through reproducible criteria, and the ratio of six practitioners to 16 general practices is similar to the national ratio of acupuncturists registered with the British Acupuncture Council to general practices. All these factors enhance the relevance of the results by replicating conditions under which an acupuncture service might be offered and used in NHS primary care.

Recruitment to the trial suggests that general practitioners are able to identify suitable patients for referral. In a retrospective survey, participating general practitioners estimated that they had identified about half the eligible patients seen in the recruiting period. No evidence was found of reluctance by general practitioners to refer patients for acupuncture. The results from this trial on the safety and possible benefits of acupuncture care for low back pain will be of interest to general practitioners who are asked about such treatment by their patients, and to patients considering acupuncture care outside the NHS. However, the generalisability of our findings rests on the assumption that participating acupuncturists, general practitioners, and patients in York are similar to those found elsewhere.

Studies of interventions for low back pain are particularly susceptible to effects arising from a regression to the mean; patients will tend to seek help at the point when the pain is at its worst or least bearable, and the clinical course of the condition is that the pain will reduce substantially for most people, with or without treatment. Commentators have indicated a need for more evidence of longer term effects, particularly related to discrete, short term interventions delivered early in an episode of persistent low back pain, that result in a reduction in recurrence and persistence of symptoms. ${ }^{30}$ Our study contributes evidence for a short term acupuncture intervention compared with usual general practitioner care for non-specific low back pain. Further clinical research is indicated to investigate the optimum timing for such an acupuncture treatment package, and to assess the value of repeated courses of acupuncture for patients experiencing recurrent episodes of low back pain.

To address the question of whether the clinical benefits observed justify the cost of the therapy, we report the cost effectiveness of this intervention in the accompanying economics paper. $^{8}$

We thank the patients, primary care staff, acupuncturists, and members of the trial advisory group, particularly Trevor Sheldon, Sally Bell-Sayer, and David Laverick our patient representative. (C) Crown copyright 2006.

Contributors: $\mathrm{KJT}$ was principal investigator and grant holder. She is guarantor. KJT, HMacP, MF, JB, JPN, and MJC developed the protocol, secured initial funding, and implemented the study. HMacP, LT, and JB secured funding for the 24 months' follow-up. LT recruited patients and managed the trial. MR advised and helped recruit the general practitioners. HMacP supervised the clinical intervention. KJT, MJC, and JPN carried out the statistical analysis. SJW undertook independent statistical analysis of the primary outcomes. KJT wrote the original draft with HMacP. All authors 


\section{Research}

commented on the draft and contributed to the interpretation of the findings.

Funding: This study was funded by the UK NHS Executive health technology programme. The views and opinions expressed do not necessarily reflect those of the NHS Executive. York Health Authority funded the treatment costs of the acupuncture. The National Coordinating Centre for Health Technology Assessment reviewed the protocol and the final report but had no direct involvement in the research process. KJT and JB joined the commissioning group of the National Coordinating Centre for Health Technology Assessment after funding for this study was awarded. Jon Nicholl is currently chair of the National Coordinating Centre for Health Technology Assessment commissioning group.

Competing interests: None declared.

Ethical approval: This study was approved by York research ethics committee.

1 Department of Health. The prevalence of back pain in Great Britain in 1998. London: Government Statistical Service, 1999.

2 Clinical Standards Advisory Group on Back Pain. Back pain:epidemiology and costs. London: HMSO, 1994.

3 BMJ Publishing Group. Clinical evidence. Low back pain and sciatica (chronic). Feb 2003. www.clinicalevidence.com

4 MacAuley D. Back pain and physiotherapy; NHS treatment is of little value. BMJ 2004;329:694-5.

5 Furlan AD, van Tulder, M, Cherkin D, Tsukayama H, Lao L, Koes B, et al. Acupuncture and dry-needling for low back pain: an updated systematic review within the framework of the Cochrane Collaboration. Spine 2005:30:944-63.

6 Thomas KJ, Nicholl J, Coleman P. Use and expenditure on complementary medicine in England-a population-based survey. Complement Ther Med 2001;9:1-11.

7 Thomas KJ, Coleman P, Nicholl J. Trends in access to complementary or alternative medicines via primary care in England: 1995-2001 results from a follow-up national survey. Fam Pract 2003;20:575-7.

8 Thomas KJ, Nicholl J, Coleman P. Use and expenditure on complementary medicine in England-a population-based survey. Complement Ther Med 2001;9:1-11

9 Ratcliffe J, Thomas KJ, MacPherson H, Brazier J. A randomised controlled trial of acupuncture care for persistent non-specific low back pain: cost effectiveness analysis. $B M$ 2006: $\mathrm{doi}=10.1136 / \mathrm{bmj} .38932 .806134 .7 \mathrm{C}$

10 Ware JE, Sherbourne CD. The MOS 36-item short-form health survey (SF-36). Med Care 1992;30:473-81.

11 Brazier JE, Harper R, Thomas KJ, Jones N, Underwood T. Outcome measures in primary health care: validating a new instrument. BMJ 1992;305:160-4.

\section{What is already known on this topic}

Non-specific low back pain is typically a recurrent condition associated with high health and social costs

Many people with back pain seek acupuncture treatment; however, evidence for long term effectiveness is sparse

\section{What this study adds}

Weak evidence was found of an effect of acupuncture care on non-specific low back pain at 12 months, but stronger evidence of a small benefit at 24 months

Referral to a qualified acupuncturist seems safe and acceptable to patients with low back pain
12 Bronfort G, Bouter LM. Responsiveness of general health status in chronic low back pain: a comparison of the COOP charts and the SF-36. Pain 1999;83):201-9.

13 Fairbank J, Davies J, Coupar J, O'Brien JP. The Oswestry low back pain disability questionnaire. Physiotherapy 1980;66:271-3.

14 Melzack R. The short-form McGill pain questionnaire. Pain 1987;30:191-7.

15 Deyo R, Cherkin D, Franklin G, Nichols JC. User's manual:low back pain type specification. Bloomington, MN: Health Outcomes Institute, 1992.

16 MacPherson H, Gould AJ, Fitter M. Acupuncture for low back pain: results of a pilot study for a randomised controlled trial. Complement Ther Med 1999;7:83-90.

17 Lee KJ, Thompson SG. Clustering by health professional in individually randomised trials. BMJ 2005;330:142-4.

18 MacPherson H, Thorpe L, Thomas KJ, Campbell M. Acupuncture for low back pain: traditional diagnosis and treatment of 148 patients in a clinical trial. Complement Ther Med 2004;12:38-44.

19 Thomas KJ, MacPherson H, Thorpe L, Brazier J, Fitter M, Campbell M, et al. Longer term clinical and economic benefits of offering acupuncture care to patients with chronic low back pain. Health Technol Assess 2005;9:1-109.

20 Ware JE Jr, Snow KK, Kosinski M, Gandek B. SF-36 health survey manual and interpretation guide. Boston,: Health Institute, New England Medical Centre, 1993.

21 Angst F, Aeschlimann A, Stucki G. Smallest detectable and minimal clinically important differences of rehabilitation intervention with their implications for required sample sizes using WOMAC and SF-36 quality of life measurement instruments in patients with osteoarthritis of the lower extremities. Arthritis Rheum 2001;4:384-91.

22 Thomas KJ, Fitter MJ, Brazier JE, MacPherson H, Campbell M, Nicholl JP, et al. Longerterm clinical and economic benefits of offering acupuncture to patients with chronic low back pain assessed as suitable for primary care management. Complement Ther Med 1999;7:91-100.

23 Kaptchuk T. Powerful placebo: the dark side of the randomised controlled trial. Lancet 1998;351:1722-5.

24 MacPherson H, Mercer SW, Scullion T, Thomas KJ. Empathy, enablement, and outcome: an exploratory study on acupuncture patients' perceptions. J Altern Complement Med 2003;9:869-76.

25 Meenan R. Developing appropriate measures of the benefits of complementary and alternative medicine.J Health Serv Res Policy 2001;6:38-43.

26 MacPherson H, Thomas KJ, Walters S, Fitter M. The York acupuncture safety study: prospective survey of 34,000 treatments by traditional acupuncturists. BMJ 2001;323:486-7.

27 Dincer F, Linde K. Sham interventions in randomized clinical trials of acupuncture-a review. Complement Ther Med 2003;11:235-42.

28 Kalauokalani D, Cherkin DC, Sherman KJ, Koepsell TD, Deyo RA. Lessons from a trial of acupuncture and massage for low back pain: patient expectations and treatment effects. Spine 2000;26:1418-24.

29 Lewith GT, Hyland ME, Shaw S. Do attitudes toward and beliefs about complementary medicine affect treatment outcomes? Am J Public Health 2002;92:1604-6.

30 Thomas KJ, Fitter M. Evaluating complementary therapies for use in the NHS: 'horses for courses' 2: alternative research strategies. Complement Ther Med 1997;5:94-8.

31 Croft P, Papageorgious A, McNally R. Low back pain. In: Stevens A, Raftery J, eds Health care needs assessment; the epidemiologically-based needs assessment reviews. 2nd Series. Abingdon, Oxford: Radcliffe Medical Press, 1997.

(Accepted 3 May 2006)

doi $10.1136 /$ bmj.38878.907361.7C

School of Health and Related Research, University of Sheffield

$\mathrm{K} J$ Thomas reader

$\mathrm{L}$ Thorpe research associate

J Brazier professor

M J Campbell professor

$\mathrm{S} \mathrm{J}$ Walters senior lecturer

$\mathrm{J}$ Nicholl professor

Foundation for Traditional Chinese Medicine, York YO24 1ET

$\mathrm{H}$ MacPherson research director

$\mathrm{M}$ Fitter director

Selby and York Primary Care Trust, York

M Roman general practitioner principal

Correspondence to: H MacPherson hm18@york.ac.uk 